\title{
Perceptual slant induced through optical contact
}

\author{
Francesco Martino and Luigi Burigana \\ University of Padua, Padua, Italy
}

\begin{abstract}
Stimuli simulating a corridor with a pole inside it were presented. The positions of the ends of the pole within the ceiling and ground of the corridor were the optical contacts of concern. The main hypothesis was that a difference between the simulated distances of these optical contacts may influence the apparent slant in depth of the pole. Two experiments were conducted, with 30 and 20 participants (university students). The tasks were a choice between three alternative responses (upward, downward, or no apparent slant) in the first experiment and matching by adjustment in the second. The results supported the hypothesized dependence of apparent slant on differences in optical contacts. The results also revealed a dependence of apparent slant on the interaction between the optical tilt of the pole and its position to the right or left of the vertical median through the stimulus. Comparisons between both effects showed that they combine in a cumulative way and that the former is weightier than the latter. Further findings were a privileged association between null optical tilt of the pole and the no apparent slant response and a bias (for poles with nonnull optical tilt) in favor of the upward apparent slant response.
\end{abstract}

The main object of this study was a possible induction effect, in the sense meant, for example, by Gogel (1972). More precisely, it was a depth induction effect, since the crucial aspects that we considered in both its inducing and test components were related to perceptual depththe third dimension of perceptual space. In studying the hypothesized effect, we focused on certain positional relations between the inducing and the test components, which we will call optical contacts. The stimuli we used were static images displayed on a monitor. Figure 1 shows an example.

The inducing component for the effect was a set of four regions covered by a random checkerboard texture, as illustrated in Figure 1. We will call this the frame (in full form: spatial reference frame) in the stimulus. Three spatial structures could be distinguished for it. First, the frame was endowed with an optical structure, which was its spatial organization as a part of the pictorial stimulusthat is, the image on the monitor. This structure was 2-D in character. ${ }^{1}$ Second, the frame had a simulated structure. We produced our stimuli with computer graphics software, by defining a program so that the resulting image represented a corridor (a 3-D virtual structure). Lastly, the frame had a perceptual structure. This was the spatial organization really perceived by an observer looking at the stimulus, as we presented it in the experiments. On the frame, three definite cues to depth were in action-linear perspective, texture, and illumination perspective - which consistently supported a 3-D perceptual rendering. For this reason, we presumed that, with high probability, the perceptual structure of the frame would be $3-\mathrm{D}$ in character. Of course, there might be differences between the simulated and the perceptual structures of the frame, in spite of their common 3-D character.

The test component was a distinct linear unit, which we will call the target (as displayed on the monitor, it was red). When the frame perceptually organized as a corridor, the target might be interpreted as a pole, with a certain position and orientation inside the corridor. By the slant of a pole in the visual field, we will mean its inclination relative to the vertical in that field; more precisely, we will define it as the angle between the vertical and the projection of the pole on the sagittal plane. We will use positive numbers for upward slants (the frontal plane containing the upper end of the pole is farther from the observer than that containing the lower end), negative numbers for downward slants (the two frontal planes stand in the opposite relation), and the number zero for no slant (the pole lies in a frontal plane). Now, regarding the target in a visual context like that shown in Figure 1, we were specifically interested in its perceptual slant - that is, its apparent inclination in depth when it was perceptually interpreted as a pole inside a corridor. In its optical specification, the target was a straight-linear component having uniform thickness and color over its extent. Thus, so far as we consider the optical properties strictly internal to the target, there seems to have been no stimulus support for a nonnull apparent slant of the target itself. Hence, if any nonnull perceptual slant was consistently proven by experiment, we would be led to conclude that

L. Burigana, luigi.burigana@unipd.it 


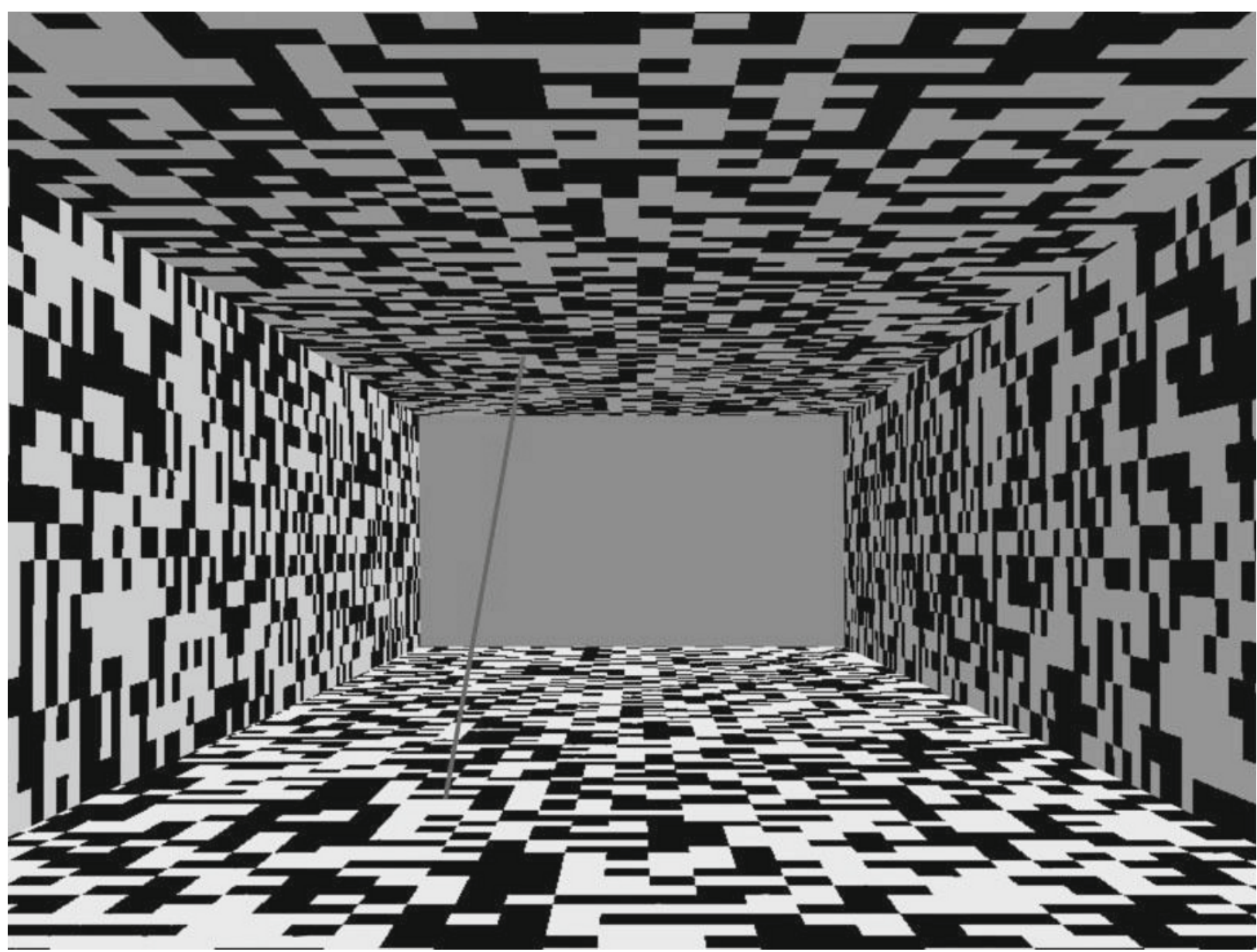

Figure 1. One of the stimuli used in our experiments. Texture elements covering the four regions were in achromatic colors (shades of gray). The oblique linear unit was red in color.

this was chiefly due to the interaction between target and frame-more precisely, to the action of the 3-D perceptual organization of the frame on the apparent inclination in depth of the target. This is the core of the depth induction effect that we surmise.

Within the kind of stimulus illustrated by Figure 1, two places were given special consideration in our analysis. They were particle $U$ of the upper region of the frame that immediately surrounded the upper end of the target and particle $L$ of the lower region, analogously defined with respect to the lower end of the target. We will call $U$ and $L$ the upper and lower locus of optical contact, respectively. ${ }^{2}$ For both loci, three distances could be distinguished. For example, relative to upper locus $U$, there was an optical distance, which was its distance from the upper edge of the frame on the image plane; a simulated distance, which was its distance from the upper edge within the simulated structure of the frame (thus, a distance in depth); and a perceptual distance, which was defined analogously, but referring to the perceptual structure of the frame. These concepts can be similarly defined for the lower locus $L$ of optical contact, by referring to the lower edge of the frame. The three kinds of distance generally differ from one another in their values in the same visual context. Nevertheless, positive correlations may be presumed between them: Simulated distance is positively related to optical distance (by geometrical rules of optical projections) and, in turn, perceptual distance is positively related to simulated distance (because of the depth cues introduced in constructing the stimuli). In particular, we considered the simulated distances of both loci of optical contact, denoted by $S D(U)$ and $S D(L)$. Their difference $S D(U)-$ $S D(L)$ was the main independent variable in our experiments. We will call it the vertical unbalance in the stimulus (considering the association between simulated and optical distances, the latter being measured on the vertical of the 2-D stimulus). For example, in the stimulus shown in Figure 1, the vertical unbalance of the target is a positive measure, since $S D(U)$ (the simulated distance of the upper optical contact) is greater than $S D(L)$ (the simulated distance of the lower optical contact).

The main experimental expectation of our research was a monotone association between vertical unbalance and perceptual slant of the target. Should this expectation be supported by the data, we would interpret it as evidence of a definite psychophysical hypothesis, the hypothesis of 
contact preservation. The meanings of such an expectation and its possible interpretation are as follows. Let us presume that $P_{1}, \ldots, P_{m}$ are stimuli of the kind illustrated in Figure 1 and $V\left(P_{1}\right), \ldots, V\left(P_{m}\right)$ are the values of vertical unbalance $V=S D(U)-S D(L)$ in them. Let us also presume that these stimuli are used in a psychophysical experiment and that, on the basis of participants' answers, we derive estimates $S\left(P_{1}\right), \ldots, S\left(P_{m}\right)$ of perceptual slant $S$ of the target, these being positive, null, or negative numbers depending on whether the perceived slant is (on average) upward, null, or downward. It may happen that, between these two sets of measures, the following relation holds true:

$$
\begin{aligned}
& V\left(P_{i}\right)<V\left(P_{j}\right) \text { if and only if } \\
& S\left(P_{i}\right)<S\left(P_{j}\right), \text { for all } 1 \leq i, j \leq m .
\end{aligned}
$$

This is what we call a monotone association between vertical unbalance $V$ (a property of the stimulus) and apparent slant $S$ (a property of the perceptual result). It means that, as the difference between the simulated distances of the upper and lower loci of optical contact increases (from negative to positive values), there is a corresponding increase in the apparent slant of the target (from downward to upward modes of slant in depth). The hypothesis we will consider in interpreting this possible experimental result is the following: In the stimulus situations used in our experiments, there was the tendency of the upper and lower ends of the target to be perceived as adjacent or attached to the upper and lower surfaces, respectively, of the frame, when this perceptually organized as a 3-D structure (an apparent corridor). We will call it the hypothesis of contact preservation, because it means that, in the process of percept formation, both loci of optical contact of the target with the upper and lower regions of the 2-D frame tend to confirm themselves as loci of perceptual contact of the target (the apparent pole) with the upper and lower surfaces of the 3-D frame (the apparent corridor). It can easily be seen, by a geometrical argument, that this hypothesis may explain the monotone association between vertical unbalance and perceptual slant of the target that we expected from our experiments.

These being the intentions, our contribution may be linked to the tradition of studies on propagation of depth information in visual perception. In this regard, a classic reference is the ground theory of space perception of Gibson (1950). This theory includes, as an essential component, a postulate that is analogous to the hypothesis of contact preservation: "Objects are represented as if resting on or attached to a surface. ... Other things being equal, two optically adjacent surfaces tend to be adjacent in depth" (Gibson, 1950, pp. 177, 180). In recent years, a fresh stream of studies has appeared on this subject, in which the initial ideas have been expanded in several respects: Not only the ground surface (the terrain), but also other surfaces variously located and oriented may serve as the inducing component in perceptual depth propagation (cf. Bian, Braunstein, \& Andersen, 2006; Meng \& Sedgwick, 2002; Sauer, Saidpour, Braunstein, \& Andersen, 2001); several kinds of adjacency or proximity relations between parts of the image (the pictorial stimulus) may intervene as the mediators in the propagation process (cf. Albert, 1999; Reinhardt-Rutland, 1999; Sauer, Braunstein, Saidpour, \& Andersen, 2002); the process may involve more than two parts in the image that, in terms of optical contact, are linked to form chains or hierarchies (nested contact relations; cf. Meng \& Sedgwick, 2001, 2002; Ni, Braunstein, \& Andersen, 2005). The hypothesis of contact preservation may be viewed as a natural theoretical component in these developments and has received support in corresponding experimental studies.

Within this area of experimental research, the most specific reference for our study is an article by Bian, Braunstein, and Andersen (2005) about the ground dominance effect - that is, the possibility that, when a target is in optical contact with both a ground and a ceiling surface but these optical contacts cannot both be confirmed as perceptual contacts (presuming that the target shows no slant in its perceptual appearance), the optical contact with the ground surface tends to prevail; that is, it is the optical contact with the ground that becomes confirmed as a perceptual contact, thus conditioning the apparent position of the target in the third dimension. However, in introducing their Experiment 4, Bian et al. (2005) also considered an alternative perceptual solution to such "contradictory layout information": It may happen that both optical contacts are confirmed as perceptual contacts, at the expense of the perceptual verticality of the target; the target may be seen as slanted in depth, in the upward or downward mode, depending on the sign of the difference between the optical contacts. This possibility precisely corresponds to the main expectation of our study, as stated above. In their Experiments 4 and 5, Bian et al. (2005) did not find convincing support for it; instead, they found evidence in favor of the hypothesis of ground dominance. The principal aim of our research was to test the stated expectation again, by modifying the stimuli in certain respects.

Besides vertical unbalance $V$, we considered other stimulus measures - in particular, the optical tilt of the target (denoted by $T$ ), which is the inclination of the target relative to the vertical (on the image plane) and is expressed by negative, positive, or null numbers, depending on whether the target leans to the left or the right or is vertical. In planning our first experiment, we introduced optical tilt $T$ as an independent variable on the basis of this consideration: There may be a privileged association between stimulus condition $T=0$ (the target is vertical on the image plane) and perceptual response $S=0$ (the target is judged to have no apparent slant in depth), so that, to gain clearer evidence of the effect we surmised (a dependence of $S$ on $V$ ), stimulus situations in which $T \neq 0$ should also be considered. The experimental results lent support to this conjecture and also highlighted another and unexpected effect of optical tilt, which we will interpret in terms of reverse projection in visual perception. This effect was confirmed by our second experiment and substantially enriched the initial expectations of this research: We have evidence not only of a depth induction process on apparent slant, but also of a reverse projection process regarding the same phenomenon. 


\section{EXPERIMENT 1}

The main characteristics differentiating our stimuli from those in Experiments 4 and 5 of Bian et al. (2005) were the following: The frame in the stimulus was formed of four regions, which were circularly adjacent so as to simulate a corridor; the target was a thin linear segment (i.e., a component that in its appearance was one-dimensional and devoid of internal structure); in two-thirds of the stimuli the target was shown tilted to the right or left (in the other third, the direction of the target coincided with the vertical of the frame). In choosing these characteristics, our purpose was to strengthen the frame in its unitary 3-D perceptual aspect and to weaken the target in its internal attributes possibly relevant to perceptual depth, so that the hypothesized dependence of the depth appearance of the latter on the 3-D organization of the former could be better appreciated.

Our first experiment involved four properties of the stimulus in the role of independent variables. They were vertical unbalance $V$, optical tilt $T$, horizontal position $H$ (a dichotomous variable, with $H=-1$ or 1, depending on whether the target was shown on the left or right of the median vertical of the frame), and optical length $L$ (i.e., the length of the target on the image plane). The first of these variables (vertical unbalance $V$ ) was the most important with regard to the specific aim of this experiment, since its possible relevance to response variable $S$ (the perceptual slant of the target) corresponded to the depth induction effect we intended to verify. The original reason for introducing stimulus property $T$ as a variable here is that stated above. As regards the other two stimulus properties (horizontal position $H$ and optical length $L$ ), initially we did not have any definite expectation regarding their possible efficacy; we introduced them as variables simply to create a sufficiently large set of stimuli on which to test the generality of our main hypothesis.

\section{Method}

Participants. The experiment was carried out by 30 participants, who took part in it voluntarily. They were undergraduate or graduate students of several faculties at the University of Padua (Italy), ranging in age from 21 to 30 years $(M=23.91), 16$ women and 14 men. All had normal or corrected-to-normal visual acuity and were unaware of the hypotheses of our study.

Stimuli. We used 90 stimuli, obtained by combining five levels of factor $V$, three of $T$, three of $L$, and two of $H$. The five levels of factor $V$ are denoted by $-2,-1,0,1$, and 2 and were specified so as to produce values of $-65^{\circ},-45^{\circ}, 0^{\circ}, 45^{\circ}$, and $65^{\circ}$ as the simulated slant of the target [simulated slant as a direct function of the simulated distances $S D(U)$ and $S D(L)$ of both loci of optical contact]. The three levels of factor $T$ are denoted by $-1,0$, and 1 and correspond to tilts of $-10^{\circ}\left(10^{\circ}\right.$ to the left), $0^{\circ}$ (vertical position), and $10^{\circ}\left(10^{\circ}\right.$ to the right). The three levels of factor $L$ are denoted by 1,2 , and 3 and correspond to lengths of $12.8,14.2$, and $15.6 \mathrm{~cm}$ as measured on the image plane (the screen on which the stimuli were shown). Lastly, the two levels of factor $H$ are denoted by -1 and 1, meaning that the center of the target was $5 \mathrm{~cm}$ to the left or right of the median vertical of the stimulus. For example, the stimulus shown in Figure 1 is that in which $V=2, T=1, L=2$, and $H=-1$.

The frame was the same in all 90 stimuli and was that illustrated in Figure 1. It was generated by computer graphics (3-D modeling software package, Autodesk 3DS Max 8.0) and programmed to simulate a corridor $550 \mathrm{~cm}$ wide, $300 \mathrm{~cm}$ high, and 2,120 cm deep. The simulated vantage point was at a height of $130 \mathrm{~cm}$ from the ground of the corridor and at a distance of $120 \mathrm{~cm}$ from its opening. On such a frame, there were three kinds of depth cues that supported its perceptual rendering as a 3-D structure: texture (a random checkerboard texture covering the four regions), linear perspective (which involved, in particular, the adjacency lines between regions), and illumination perspective (in the simulation, an invisible light source was located next to the top-right front corner of the corridor).

The stimuli were displayed on a 22 -in. CRT monitor with a pixel resolution of $800 \times 600$ pixels, controlled by a Windows 1998 workstation. On the monitor, the width and height of the contour of the frame were 40 and $30 \mathrm{~cm}$, respectively, and the width and height of its central region were 13.8 and $7.4 \mathrm{~cm}$, respectively. The light and dark components of the texture and the central region of the frame were of different shades of gray (the mean luminances of light components in the top, bottom, left, and right textured regions were $27.42,102.86,68.08$, and $31.45 \mathrm{~cd} / \mathrm{m}^{2}$, respectively; the mean luminances of the corresponding dark components were $1.07,1.15$, 0.96 , and $0.99 \mathrm{~cd} / \mathrm{m}^{2}$, respectively; the luminance of the central region was $28.12 \mathrm{~cd} / \mathrm{m}^{2}$ ). The target was red (luminance, $43.47 \mathrm{~cd} / \mathrm{m}^{2}$ ). The distance from the eyes to the monitor was $50 \mathrm{~cm}$. A chinrest was mounted at a position appropriate to this viewing distance. At this distance, the two external sides of the frame subtended visual angles of $43.6^{\circ}$ and $33.4^{\circ}$, and the target in its three possible lengths subtended visual angles of $14.59^{\circ}, 16.16^{\circ}$, and $17.73^{\circ}$. Vision was binocular. The experiment was run in a darkened room.

Procedure. For each participant, the experimental session began with a practice phase, the aim of which was to illustrate the kind of stimuli to be observed and how to express responses. The main point was to explain to the participants the spatial property that they were asked to judge - that is, the apparent slant in depth of the red pole in the scene, which was the inclination in the forward/backward direction, to be distinguished from the apparent tilt (inclination in the right/left direction) or other types of inclination. For this purpose, we not only presented some images on the monitor generally similar to those to be shown in the main phase, but also used a simple mechanical device in which a suspended pole was rotated in various directions by the experimenter (F.M.)

In the main phase of the session, all 90 stimuli were separately presented, in an order independently randomized for each participant. The sequence of 90 trials was split into three blocks of equal size and separated by pauses, the duration of which was chosen by the participants. In each trial, a white fixation cross $(0.5 \mathrm{sec})$ first appeared in the center of the screen, and then one of the 90 stimuli was shown for $3 \mathrm{sec}$. The observers' task was to judge whether the red pole in the scene was slanted upward, downward, or neither (it appeared lying on a frontal plane) and to express their judgment, after the image had disappeared, by pressing one of three buttons on a response box. The central button was associated with the no-slant judgment for all the participants, whereas the left and right buttons were balanced for upward slant and downward slant across participants. The experimental session, including both practice and main phases, took from 14 to $17 \mathrm{~min}$ to complete.

\section{Results}

The experiment comprised four factors $(V, T, L, H)$, response variable $S$ (which is categorical), and repeated measures (each of the 30 participants gave a response to each of the $90=5 \cdot 3 \cdot 3 \cdot 2$ experimental stimuli). We analyzed the data by fitting and interpreting a log-linear model (Agresti, 2002). First, from the data file, we obtained a five-dimensional frequency table $\left(m_{v t h s}\right)$ where, for $v \in\{-2,-1,0,1,2\}, t \in\{-1,0,1\}, l \in\{1,2,3\}$, $h \in\{-1,1\}$, and $s \in\{-1,0,1\}$, frequency $m_{v t h s}$ was the number of participants who gave response $S=s$ when presented with the stimulus in which $V=v, T=t, L=l$, 
Table 1

Maximum Likelihood Estimates of Parameters in Log-Linear Model Accepted for Data in Experiment 1

\begin{tabular}{|c|c|c|c|c|c|c|}
\hline \multicolumn{7}{|c|}{ A. Component $\lambda^{S}$} \\
\hline & $S=-1$ & $S=0$ & $S=1$ & & \multirow[b]{4}{*}{$V=2$} & \\
\hline & -0.153 & 0.163 & -0.010 & & & \\
\hline \multicolumn{6}{|c|}{ B. Component $\lambda^{V S}$} & \\
\hline & $V=-2$ & $V=-1$ & $V=0$ & $V=1$ & & \\
\hline$S=1$ & -0.456 & -0.367 & -0.045 & 0.267 & 0.601 & \\
\hline$S=0$ & -0.148 & 0.054 & 0.177 & 0.113 & -0.196 & \\
\hline$S=-1$ & 0.604 & 0.313 & -0.132 & -0.380 & -0.405 & \\
\hline \multicolumn{7}{|c|}{ C. Component $\lambda^{T S}$} \\
\hline & $T=-1$ & $T=0$ & $T=1$ & & & \\
\hline$S=1$ & 0.279 & -0.685 & 0.405 & & & \\
\hline$S=0$ & -0.562 & 1.216 & -0.653 & & & \\
\hline$S=-1$ & 0.282 & -0.531 & 0.248 & & & \\
\hline \multicolumn{7}{|c|}{ D. Component $\lambda^{T H S}$} \\
\hline & \multicolumn{3}{|c|}{$H=-1$} & \multicolumn{3}{|c|}{$H=1$} \\
\hline & $T=-1$ & $T=0$ & $T=1$ & $T=-1$ & $T=0$ & $T=1$ \\
\hline$S=1$ & -0.338 & 0.066 & 0.272 & 0.338 & -0.066 & -0.272 \\
\hline$S=0$ & 0.129 & -0.022 & -0.107 & -0.129 & 0.022 & 0.107 \\
\hline$S=-1$ & 0.209 & -0.044 & -0.165 & -0.209 & 0.044 & 0.165 \\
\hline
\end{tabular}

and $H=h$. Then we searched for a log-linear model which was optimal in simplicity and goodness of fit relative to the table $\left(m_{v t l h s}\right)$ of observed frequencies. The search was carried out in two stages. First, we searched for a hierarchical log-linear model that was optimal in the stated sense, and then we tried to simplify the optimal hierarchical log-linear model further by omitting some of its components, so far as such omissions did not entail any substantial increase in residual deviance.

The log-linear model resulting from this search is given by the following equation:

$$
\log \left(m_{v t h s}\right)=\mu+\lambda_{s}^{S}+\lambda_{v s}^{V S}+\lambda_{t s}^{T S}+\lambda_{t h s}^{T H S} .
$$

It expresses the logarithm of the observed frequencies as the sum of five components, which are intercept $\mu$ (with no useful meaning, in the present context); component $\lambda S$, directly associated with response variable $S$; components $\lambda^{V S}$ and $\lambda^{T S}$, specifying the separate actions of factors $V$ and $T$ on response $S$; and component $\lambda^{T H S}$, specifying the interaction of factors $T$ and $H$ on response $S$. The model is nonhierarchical (e.g., it has component $\lambda^{T H S}$ but not component $\lambda^{H S}$ ). Its residual deviance (likelihood-ratio statistic) is $G^{2}=200.28$, with $d f=251(=270-19$, 270 being the size of the frequency table, 19 the number of free parameters in the model), and $p=.991$ [relative to distribution $\chi^{2}(251)$ ]. The low value of residual deviance (when compared with the null deviance of our data, which is $G^{2}=1,557.86$ ) and the corresponding high value of $p$ are proof of a very good fit of the model to the data. Table 1 lists the maximum-likelihood estimates of the four classes of parameters forming the model (the estimate of the intercept is $\mu=2.029$ ).

In the next few paragraphs, we will separately comment on the components of the accepted log-linear model. For each component, we will begin by giving its specific devi- ance, with corresponding degrees of freedom and $p$ value. By the specific deviance of a component, we mean the difference between the residual deviance (likelihood-ratio statistic) of the log-linear model when it is deprived of that component and the residual deviance of the log-linear model defined above (i.e., including the component). This difference is a measure of the loss of goodness of fit that would derive from leaving out that specific component, so that it is an index of the "right" of the component itself to be a member of the model accepted for the data.

Component $\lambda^{S}$ (specific deviance $G^{2}=95.236, d f=2$, $p<.001)$ means that, in the whole set of 2,700 = 90 30 responses by participants, alternatives $-1,0$, and 1 were not of equivalent presence. More precisely, Table 1A shows that the most preferred response was $S=0$ (no apparent slant in depth), then the $S=1$ response (the pole appears slanted upward), and, last, the $S=-1$ response (the pole appears slanted downward). This order can also be directly seen on the collapsed frequencies associated with the three alternatives, which are 1,051 for $S=0,899$ for $S=1$, and 750 for $S=-1$. Unexpected, in particular, was the difference in frequency between alternatives $S=1$ and $S=-1$ (significant to the binomial test with $p<.001$ ), which revealed a bias in favor of the upward slant response.

Component $\lambda^{V S}$ (specific deviance $G^{2}=568.225$, $d f=8, p<.001)$ means that there is a strong association between stimulus variable $V$ and response variable $S$. Table $1 \mathrm{~B}$ shows that this is an increasing monotone association, when ranges $\{-2,-1,0,1,2\}$ and $\{-1,0,1\}$ of the two variables are ordered in the natural way. In more detail, the table shows that, as variable $V$ increases over its range, the chance of an $S=1$ response increases, that of an $S=$ -1 response decreases, and that of an $S=0$ response first increases and then decreases. This sequence was precisely what we expected on the basis of our main hypothesis, con- 
Table 2

Frequencies of Responses $S=-1, S=0$, and $S=1$ to Stimuli With Different Values of Property $V$ (Vertical Unbalance of Target) in Experiment 1

\begin{tabular}{lccccr}
\hline & $V=-2$ & $V=-1$ & $V=0$ & $V=1$ & $V=2$ \\
\hline$S=1$ & 107 & 120 & 164 & 217 & 291 \\
$S=0$ & 180 & 226 & 253 & 230 & 162 \\
$S=-1$ & 253 & 194 & 123 & 93 & 87 \\
\hline
\end{tabular}

cerning the dependence of the apparent slant of the target on the vertical unbalance in the stimulus. The same course can be directly seen in Table 2, which shows the collapsed frequencies on the pair of dimensions $(V, S)$.

Component $\lambda^{T S}$ (specific deviance $G^{2}=1,975.407$, $d f=4, p<.001)$ signifies quite a strong connection between stimulus variable $T$ and response variable $S$. The scheme, as revealed by the values in Table $1 \mathrm{C}$, is as follows: In the $T=0$ condition (the target was upright in the 2-D stimulus), there was a clear-cut dominance of the $S=0$ response (the target was judged to have no slant in the 3-D perceptual scene), whereas in the $T=-1$ and $T=1$ conditions, the chance of the $S=0$ response was greatly diminished and there was an increase in the chance of both the $S=-1$ and $S=1$ responses. The same scheme is directly seen in Table 3 , which shows the frequencies collapsed on the pair of dimensions $(T, S)$ (note, in this table, that in both the $T=-1$ and $T=1$ conditions, the increase in frequency is greater for the $S=1$ response than for the $S=-1$ response, which accords with the bias in favor of the upward slant response when $T \neq 0$, noted above). Thus, there was a strong privileged association between stimulus condition $T=0$ and the $S=0$ response. This response prevailed in general, as was pointed out when component $\lambda^{S}$ was discussed, but its prevalence became much greater in stimulus condition $T=0$. We will add to this point in the Discussion section.

Component $\lambda^{T H S}$ (specific deviance $G^{2}=195.022$, $d f=4, p<.001)$ proves the existence of an interaction of stimulus variables $T$ and $H$ on response variable $S$. Actually, the values in Table 1D show that, when $H=-1$ (target in left visual hemifield), the $T=-1$ condition favored the $S=-1$ response and the $T=1$ condition the $S=1$ response, whereas when $H=1$ (target in right visual hemifield), the opposite was the case - that is, the $T=-1$ condition favored the $S=1$ response and the $T=1$ condition the $S=-1$ response. The same configuration can be seen in Table 4, which lists the frequencies collapsed on the triple of dimensions $(T, H, S)$. This interaction does not correspond to a hypothesis conceived in planning the experiment, yet it may be given a plausible interpretation in geometric-optical terms. Indeed, let us presume that a linear object (a pole) is given in physical 3-D space so that its tilt relative to the vertical is null (i.e., the pole is parallel to the sagittal plane). A simple argument shows that, when the object lies in the left hemifield (i.e., $H=-1$ ), its polar projection on the frontal plane passing through the vantage point is tilted left $(T<0)$ or right $(T>0)$, depending on whether the object is physically slanted downward $(S<0)$ or upward $(S>0)$. The same argument shows that, when the object lies instead in the right hemifield (i.e., $H=1)$, its polar projection is tilted left $(T<0)$ or right $(T>0)$, depending on whether the object is physically slanted upward $(S>0)$ or downward $(S<0)$. Thus, the following relational constraint on variables $T, H, S$ is true at the optical level:

if $H=-1$, then $T<0$ or $T>0$, depending on whether $S<0$ or $S>0$;

if $H=1$, then $T<0$ or $T>0$, depending on whether $S>0$ or $S<0$.

Now, let us turn this optical constraint into a (conjectural) psychophysical constraint, simply by interpreting $S$ as the perceived (rather than the physical) slant of the object. The rule then allows us to predict that, if $H=-1$, the $T<0$ and $T>0$ stimulus conditions favor the $S<0$ and $S>0$ responses, respectively, whereas if $H=1$, the $T<0$ and $T>0$ stimulus conditions favor the $S>0$ and $S<0$ responses, respectively. But this prediction exactly fits the configuration shown by the parameter estimates in Table 1D and the response frequencies in Table 4. We will call the effect revealed by these tables the reverse projection process on perceptual slant, because the way we interpret it matches the reverse projection heuristic sometimes used in discussing visual phenomena (cf. Johansson, 1970, p. 70; Sedgwick, 2001, p. 130).

There are five variables in our data set, so that the saturated log-linear model fitting the data has $2^{5}=32$ components. We found a well-fitting model comprising only 5 of these components, and this may be viewed as a success in simplicity. In particular, neither component $\lambda^{V T S}$ (interaction effects of $V$ and $T$ on $S$ ) nor component $\lambda^{V T H S}$ (interaction effects of $V, T$, and $H$ on $S$ ) are accepted in the model; specific deviances are $G^{2}=18.671(d f=16, p=.286)$ for the former, and $G^{2}=22.985(d f=16, p=.114)$ for the latter. We note this circumstance because, as we shall see, these are two points on which there is a difference between the results of our experiments.

\section{EXPERIMENT 2}

The stimuli in Experiment 2 were similar to those in Experiment 1 . The plan differed from Experiment 1 in three respects, two of which concerned the experimental factors and one the experimental task. On the one hand, variable $L$ (the optical length of the target) was removed from the set of experimental factors, which means that all the stimuli in the new experiment were equivalent in that characteristic. We made this simplification by also considering that

Table 3

Frequencies of Responses $S=-1, S=0$, and $S=1$ to Stimuli With Different Values of Property $T$ (Pictorial Tilt of Target) in Experiment 1

\begin{tabular}{lcrc}
\hline & $T=-1$ & $T=0$ & $T=1$ \\
\hline$S=1$ & 383 & 95 & 421 \\
$S=0$ & 181 & 708 & 162 \\
$S=-1$ & 336 & 97 & 317 \\
\hline
\end{tabular}


Table 4

Frequencies of Responses $S=-1, S=0$, and $S=1$ to Stimuli With Different Values of Properties $T$ and $H$ (Pictorial Tilt and Horizontal Position of Target) in Experiment 1

\begin{tabular}{lcrrrrrrr}
\hline & \multicolumn{3}{c}{$H=-1$} & & \multicolumn{3}{c}{$H=1$} \\
\cline { 2 - 4 } \cline { 7 - 8 } \cline { 6 - 8 } & $T=-1$ & $T=0$ & $T=1$ & & $T=-1$ & $T=0$ & $T=1$ \\
\hline$S=1$ & 142 & 54 & 263 & & 241 & 41 & 158 \\
$S=0$ & 103 & 349 & 66 & & 78 & 359 & 96 \\
$S=-1$ & 205 & 47 & 121 & & 131 & 50 & 196 \\
\hline
\end{tabular}

the data in Experiment 1 showed no involvement of variable $L$ in any significant effect on the perceptual property under study. On the other hand, the number of levels of factor $T$ (the optical tilt of the target) was increased, from three to five. The data in Experiment 1 showed that factor $T$ played an important role in conditioning the response variable, so that we judged it worthy of closer scrutiny. The task for the participants in Experiment 2 was matching by adjustment, rather than a categorical judgment, as in Experiment 1. Our aim was thus to obtain more precise information-quantitative rather than classificatoryconcerning the apparent slant of the target in the scene.

On the basis of measures produced through matching by adjustment, the aims of Experiment 2 were as follows: to confirm and specify better the dependence of apparent slant $S$ on vertical unbalance $V$ of the target (what we will call the depth induction process); to confirm and specify better the dependence of $S$ on pictorial tilt $T$ of the target, both in terms of main effects of $T$ and in terms of interaction effects of $T$ and $H$ (what we will call the reverse projection process); and to explore the interaction between both processes - in particular, to appreciate their relative importance in conditioning the apparent slant of the target.

\section{Method}

Participants. The experiment was carried out by 20 subjects ( 9 women, $11 \mathrm{men}$ ), whose general characteristics were similar to those of the participants in Experiment 1. Eight of them had also taken part in Experiment 1, about 3 months previously.

Stimuli. Fifty stimuli were produced by combining five levels of factor $V$, five of factor $T$, and two of factor $H$ (the horizontal position of the target). The levels of $V$ and $H$ are labeled as in Experiment 1 and correspond to the same measures defined above. The levels of $T$ are denoted by $-2,-1,0,1$, and 2 and correspond to measures $-15^{\circ},-5^{\circ}$ (tilts of the target to the left), $0^{\circ}$ (no tilt), and $5^{\circ}$ and $15^{\circ}$ (tilts of the target to the right). The optical length of the target, as measured on the monitor, was kept constant $(14.2 \mathrm{~cm})$ across the stimuli. The distance from the eyes to the monitor was $74 \mathrm{~cm}$. At this distance, the visual angle subtended by the target was $10.96^{\circ}$.

All other conditions - the geometric, photometric, and simulated properties of the frame, color of the target, properties of the monitor, position of the observer relative to the monitor and so forth-were the same as those in Experiment 1.

Procedure. The mechanical device for the adjustment task was a ball-and-socket joint. The socket-a truncated hollow sphere-was fixed through a pedestal to the table at which the participant was sitting. A pole $5 \mathrm{~mm}$ in diameter, $20 \mathrm{~cm}$ in length, and red in color was joined to the ball and could be rotated by hand in all directions (maximum of $86^{\circ}$ from the vertical). At the base and vertex of the pole were two markers that reflected rays coming from three infrared cameras (ELITE motion analysis system, Bioengineering Technology \& Systems), these being placed in three of the upper corners of the experiment room. The cameras were linked to the workstation used to control the experiment, and the integrated system was equipped so as to compute and record the 3-D orientation of the adjustable pole at any given moment in the experimental session. For our purposes, the orientation was expressed as a pair of angular measures, which were slant (with respect to the vertical, as defined in the introduction) and tilt (i.e., the tilt of the orthogonal projection of the pole on the frontal plane). The device for adjustment was placed in front of the participant, $24 \mathrm{~cm}$ from the chinrest and at a height so that the vertex of the pole (when it stood vertically) was $17 \mathrm{~cm}$ below the line of sight (from the eye to the center of the monitor). On both sides of the device, at a distance of $45 \mathrm{~cm}$, were two lamps that selectively lighted up the pole when the participant was performing the adjustment task. Apart from these lamps and the monitor, there was no other source of light to the participant in the experiment room. The setup is sketched in Figure 2.

The organization of the experimental session-practice and main phases, randomized order of stimuli, a pause halfway through the series of stimuli, and so forth-was similar to that in Experiment 1 . For each participant, the main phase was a series of 50 trials, 1 for each of the stimuli. In each trial, after a signal on the monitor (a white fixation cross, $0.5 \mathrm{sec}$ ), a stimulus was shown for $5 \mathrm{sec}$. As the stimulus disappeared, the side lamps were automatically turned on, and the participants had to adjust the pole of the device to match the orientation of the pole in the scene that they had just seen on the monitor - that is, to adjust the direction of the mechanical pole so that it was 3-D parallel to the pole in the scene. This done, they pressed the space bar on a computer keyboard, so that the orientation of the adjusted pole could be computed and recorded. The session took from 40 to $45 \mathrm{~min}$ to complete.

\section{Results}

The data considered were those on stimulus variables $V$, $T, H$, and response variable $S$, which, in Experiment 2, was the matched slant of the adjustable pole - that is, the slant of the pole of the mechanical device as it was computed at the end of each adjustment operation. On these data, we ran a repeated measures three-way ANOVA, which yielded the results shown in Table 5 .

Thus, five of the seven possible effects of the factors on response variable $S$ proved to be significantly nonnull (at a significance level of .001). To illustrate the first four of these effects, we refer to Figure 3, which was obtained by plotting the specific means of variable $S$ against the levels of factor $V$ (panel A), the levels of factor $T$ (panel B), the combined levels of factors $V$ and $T$ (panel C), and the combined levels of factors $T$ and $H$ (panel D).

Figure 3A shows that, between response variable $S$ and stimulus variable $V$, there is an increasing monotone dependence: As $V$ increases over its range $\{-2,-1,0$, $1,2\}$ there is a corresponding increase in $S$, from negative (downward slant) to positive (upward slant). This dependence fits that found in the data in Experiment 1 (see Table 2), and both of them support the main claim of our study - that is, a monotone association between vertical unbalance and perceptual slant of the target. Figure 3B shows two aspects of the relation between stimulus variable $T$ and response variable $S$. One is that, among the values of $T$, value $T=0$ was the one that mostly favored a response close to $S=0$; that is, the adjustable pole was set perpendicular to the line of sight. This finding corresponds to the privileged association between the $T=0$ condition and the $S=0$ response noted in discussing component $\lambda^{T S}$ of the log-linear model for Experiment 1. 


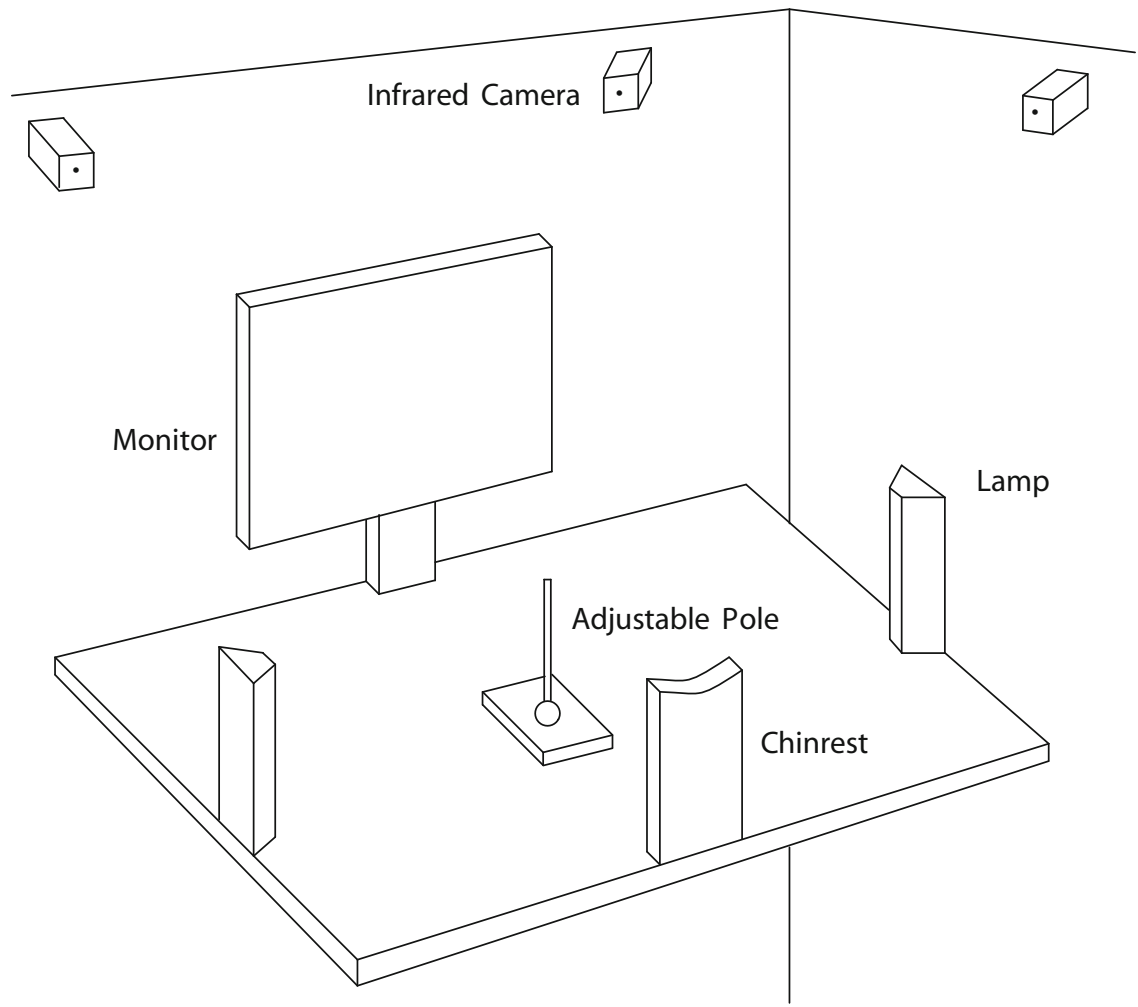

Figure 2. Setup of Experiment 2, with monitor, device for adjustment, and infrared cameras for capturing orientations of an adjustable pole.

The other aspect is that, when $T \neq 0$, the matched slant moved toward positive (rather than negative) values, so that, on the whole, the upward direction of matched slant prevailed. This aspect too has an analogue in the results of Experiment 1 (we noted it in discussing component $\lambda S$ of the log-linear model and in commenting on Table 3). Figure $3 \mathrm{C}$ represents 15 means of variable $S$, corresponding to subsets of stimuli distinguished by the levels of factor $V$ and the $T=0,(T=-1$ or $T=1)$, and $(T=-2$ or $T=2)$ conditions on factor $T$ (we apply this simplification, because diagrams of means for $T=-1$ and $T=1$ run quite close to each other, and the same is true of $T=-2$ and $T=2$ ). The figure shows that the diagram relating the means of $S$ to the levels of $V$ is steeper in the $(T=-2$ or $T=2$ ) condition than in the $T=0$ condition, which plausibly signifies that the dependence of $S$ on $V$ is stronger in the former condition than in the latter. This finding supports one idea we considered when planning our experiments - that is, that the hypothesized dependence of perceptual slant on vertical unbalance would better appear in stimulus situations in which the target was optically tilted (the idea was not convincingly supported by Experiment 1 , since component $\lambda^{V T S}$ failed to be accepted in the log-linear model). Lastly, Figure 3D shows that there were two circumstances in which the tendency to set the pole in the upward direction became reinforced: when $H=-1$ and $T>0$ (i.e., the target was in the left visual hemifield and tilted to the right in the stimulus) and when $H=1$ and $T<0$ (i.e., the target was in the right hemifield and tilted to the left). These associations fit the psychophysical relational constraint described in commenting on component $\lambda^{T H S}$ in the log-linear model and may be taken as further proof of the intervention of a reverse projection process conditioning perceptual slant.

The fifth significant source of variability in Table 5 is the interaction of factors $V, T$, and $H$ on response variable $S$ (this is another difference between the results of Experiment 2 and those of Experiment 1, since component $\lambda$ VTHS

Table 5

Statistics Resulting From Repeated Measures Three-Way ANOVA Run on Data in

Experiment 2 (Response Variable Is Matched Slant $S$ )

\begin{tabular}{lrrrr}
\hline \multicolumn{1}{c}{ Source } & \multicolumn{1}{c}{$\mathrm{SS}$} & $d f$ & $F$ & \multicolumn{1}{c}{$p$} \\
\hline$V$ & $66,418.54$ & 4,76 & 27.567 & $3.77 \cdot 10^{-14}$ \\
Error & $45,776.77$ & & & \\
$T$ & $4,610.61$ & 4,76 & 7.771 & $2.64 \cdot 10^{-5}$ \\
Error & $11,272.36$ & & & \\
$H$ & 0.97 & 1,19 & 0.008 & .929 \\
Error & $2,385.47$ & & & \\
$V \times T$ & $3,680.77$ & 16,304 & 4.299 & $1.38 \cdot 10^{-7}$ \\
Error & $16,265.82$ & & & \\
$V \times H$ & $1,067.95$ & 4,76 & 3.324 & \\
Error & $6,103.56$ & & & \\
$T \times H$ & $10,098.26$ & 4,76 & 16.375 & $1.03 \cdot 10^{-9}$ \\
Error & $11,717.27$ & & & \\
$V \times T \times H$ & $3,489.24$ & 16,304 & 3.065 & $8.16 \cdot 10^{-5}$ \\
Error & $21,627.08$ & & & \\
\hline
\end{tabular}


A
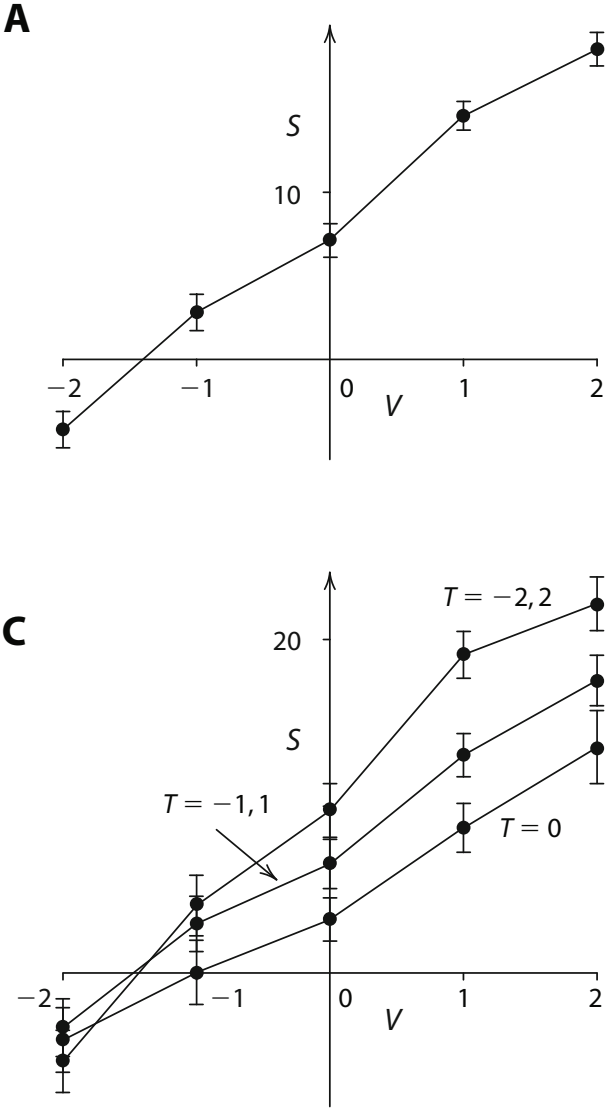

B
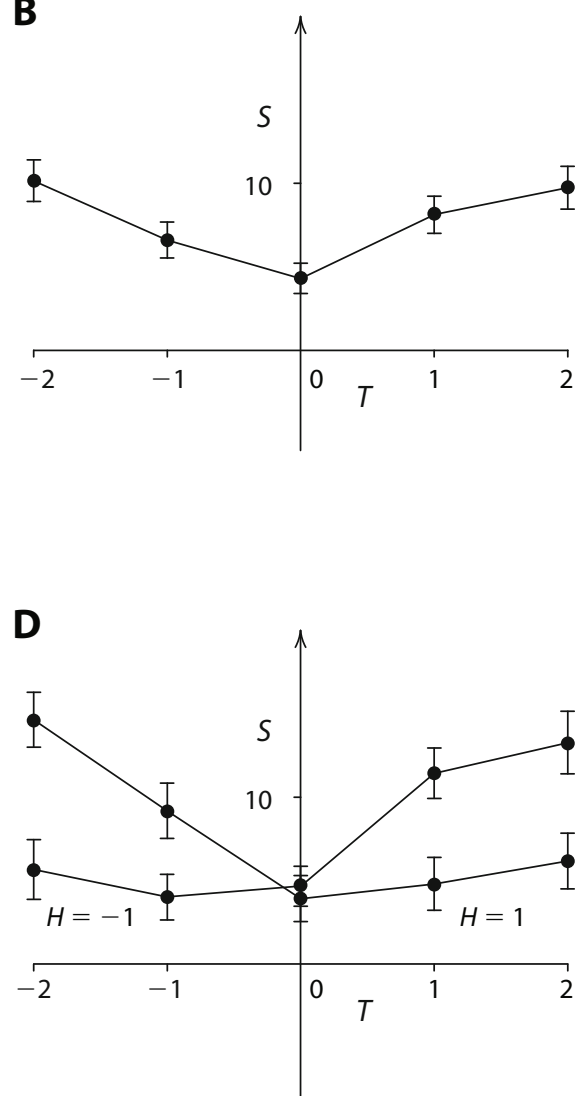

Figure 3. Plots of means of matched slant $S$ corresponding to levels of $V(A)$, levels of $T$ (B), combined levels of $V$ and $T(C)$, and combined levels of $T$ and $H(D)$, in Experiment 2. Matched slant (ordinate) is measured in degrees. Positive versus negative values represent upward versus downward slant. The length of vertical bars is twice the standard error of the corresponding mean.

was not accepted in the log-linear model). The three-way interaction properly concerns a system of $50=5 \cdot 5 \cdot 2$ means - that is, as many means as are the combinations of levels of factors $V, T$, and $H$ (and the stimuli in the experiment). Here, we will not try to interpret that interaction by examining the whole system of means. Instead, we will extract a simpler but well-balanced subsystem and examine it so as to look into the way in which the depth induction process interacts with the reverse projection process in conditioning the perceptual slant of the target.

The set of 50 stimuli may be partitioned into set $A_{\mathrm{d}}$ of 20 stimuli in which $V<0$, set $A_{\mathrm{v}}$ of 10 stimuli in which $V=0$, and set $A_{\mathrm{u}}$ of 20 stimuli in which $V>0$ (in this notation, subscripts $\mathrm{d}, \mathrm{v}$, and $\mathrm{u}$ are used as the initials of words downward, vertical, and upward, the second being equivalent to no slant). The same set of 50 stimuli may also be partitioned into set $B_{\mathrm{d}}$ of 20 stimuli in which either $(H=-1$ and $T<0)$ or $(H=1$ and $T>0)$, set $B_{\mathrm{v}}$ of 10 stimuli in which $T=0$, and set $B_{\mathrm{u}}$ of 20 stimuli in which either $(H=-1$ and $T>0)$ or $(H=1$ and $T<0)$. Now, the stimuli in $A_{\mathrm{d}}, A_{\mathrm{v}}$, and $A_{\mathrm{u}}$ are those in which, by the depth induction process, downward slant $(S<0)$, no-slant $(S=0)$, and upward slant $(S>0)$ responses are favored, respectively. The same may be said of stimuli in $B_{\mathrm{d}}, B_{\mathrm{v}}$, and
$B_{\mathrm{u}}$, but with reference to the reverse projection process. Of special interest are some intersections between these sets: The stimuli in $A_{\mathrm{d}} \cap B_{\mathrm{d}}$ (respectively, in $A_{\mathrm{u}} \cap B_{\mathrm{u}}$ ) are those in which both of the two processes support a downward (respectively, upward) response, whereas the stimuli in $A_{\mathrm{d}} \cap B_{\mathrm{u}}$ and $A_{\mathrm{u}} \cap B_{\mathrm{d}}$ are those in which the two processes do not match, since for any stimulus in $A_{\mathrm{d}} \cap B_{\mathrm{u}}$, the depth induction process supports a downward response and the reverse projection process an upward one, and the opposite is true for any stimulus in $A_{\mathrm{u}} \cap B_{\mathrm{d}}$.

Table 6 lists the means and standard deviations of the response variable (matched slant) for 8 of the 50 stimuli in the experiment (each statistic was computed on 20 measures, which were the slants matched by the participants for one of the stimuli). The 8 stimuli are representative, since they are extreme in the four intersected blocks. Specifically, the $(H=-1, T=-2, V=-2)$ and $(H=1$, $T=2, V=-2)$ stimuli are extreme in block $A_{\mathrm{d}} \cap B_{\mathrm{d}}$, the $(H=-1, T=2, V=-2)$ and $(H=1, T=-2, V=-2)$ stimuli in block $A_{\mathrm{d}} \cap B_{\mathrm{u}}$, the $(H=-1, T=-2, V=2)$ and $(H=1, T=2, V=2)$ stimuli in block $A_{\mathrm{u}} \cap B_{\mathrm{d}}$, and the $(H=-1, T=2, V=2)$ and $(H=1, T=-2, V=2)$ stimuli in block $A_{\mathrm{u}} \cap B_{\mathrm{u}}$. Two aspects of the table deserve comment. One is that, on both the left and right parts of the 
Table 6

Means and Standard Deviations for Matched Slants (Measured in Degrees) for 8 of 50 Stimuli in Experiment 2

\begin{tabular}{|c|c|c|c|c|c|c|c|c|}
\hline & \multicolumn{4}{|c|}{$H=-1$} & \multicolumn{4}{|c|}{$H=1$} \\
\hline & \multicolumn{2}{|c|}{$V=-2$} & \multicolumn{2}{|c|}{$V=2$} & \multicolumn{2}{|c|}{$V=-2$} & \multicolumn{2}{|c|}{$V=2$} \\
\hline & $M$ & $S D$ & $M$ & $S D$ & $M$ & $S D$ & $M$ & $S D$ \\
\hline$T=2$ & -6.053 & 20.604 & 24.308 & 14.065 & -4.991 & 16.043 & 20.642 & 13.206 \\
\hline$T=-2$ & -9.321 & 16.256 & 16.734 & 16.569 & -0.774 & 14.046 & 26.847 & 12.599 \\
\hline
\end{tabular}

table, the means that are extreme (either negative or positive) correspond to stimuli in which depth induction and reverse projection processes match each other in supporting a certain direction of perceptual slant. For example, on the left part, the extreme negative mean is that for stimulus ( $H=-1, T=-2, V=-2)$, which belongs to block $A_{\mathrm{d}} \cap B_{\mathrm{d}}$ (both processes support a downward perceptual slant), and the extreme positive mean is that for stimulus $(H=-1, T=2, V=2)$, which belongs to block $A_{\mathrm{u}} \cap B_{\mathrm{u}}$ (both processes support an upward perceptual slant). A similar scheme may be seen on the right part of the table. This configuration may be interpreted as proof that the two processes interact by accumulating their effects on the resulting perceptual property (the apparent slant of the target): When the predicted effects of both processes match, the resulting property is more extreme in value (than when their predicted effects do not match), and the value accords in direction with the predicted effects (cf. Bülthoff $\&$ Mallot, 1990, p. 122, about accumulation as a form of interaction between perceptual factors). The other aspect deserving comment regards differences between means in the table. Specifically, we found that the two differences of means in the same row of the left part of the table (i.e., $24.308-(-6.053)=30.361$ and $16.734-(-9.321)=$ 26.055 ) are much larger than the two differences of means in the same column of that part (i.e., $-6.053-(-9.321)=$ 3.268 and $24.308-16.734=7.574)$. This discrepancy is corroborated by the $t$ test, which yields significant results for both differences within rows $(p<.001)$, but for no difference within columns ( $p=.575$ and $p=.127)$. The same scheme holds true on the right part of the table. Now, differences within rows are those due to $V$ (the vertical unbalance of the target), which is the key factor in the depth induction process, whereas differences within columns are those due to $T$ (the optical tilt of the target), which is the key factor in the reverse projection process. Thus, the observed discrepancy between differences may be interpreted as proof that the former process is stronger than the latter in conditioning the perceptual slant of the target.

\section{DISCUSSION}

The main expectation of this research was that a difference regarding the optical contacts of a linear component (the target) with the upper and lower regions of a reference frame (this being endowed with 3-D perceptual organization) may give rise to an apparent slant in depth of the linear component itself, and that the mode of this apparent slant may monotonically depend on the sign and size of that difference. This expectation was confirmed by both experiments, in terms of the significance of component $\lambda^{V S}$ of the log-linear model fitted to the data in Experiment 1 and that of the main effects of factor $V$ on response variable $S$ in the ANOVA run on the data in Experiment 2. Thus, according to our results, the question raised by Bian et al. (2005) in introducing their Experiment 4 can be answered in the positive: Contradictory layout information concerning the extremes of an oblong component in the stimulus may perceptually result in an apparent inclination in depth of the component itself. This is not precisely what Bian et al. (2005) found in their Experiments 4 and 5 (instead, they found evidence in favor of the ground dominance effect, which was the leading theme of their study). In our view, such differences in results can be plainly understood by considering the differences in the stimuli used in our and their experiments. ${ }^{3}$ The main changes we made in constructing our stimuli have already been specified in the paragraph introducing Experiment 1. They concerned both the frame (corridor) and the target (pole) and were generally aimed at strengthening the former in its unitary 3-D perceptual aspect and weakening the latter in its internal attributes possibly relevant to perceptual depth. We conjectured that, by so doing - that is, strengthening the inducing component and weakening the test component in their spatial characteristics - the surmised depth induction effect could be brought better to light. We interpret the main results of our experiments as a proof of the soundness of this conjecture and of the real intervention - in suitable conditions - of the hypothesized induction effect.

We propose to interpret the monotone association between stimulus variable $V$ and perceptual variable $S$ as evidence for the hypothesis of contact preservation: "In the absence of information to the contrary, there is a strong tendency for optical contact to give rise to the perception of physical contact" (Sedgwick, 2001, p. 137). We propose this considering that, relative to the stimuli used in our experiments, the hypothesis above can explain, in a simple way, the monotone association expected and found in our data. Our stimuli were such that the target came into optical contact with the frame in a plain manner: The target was a continuous, uniform component in the picture, and no additional optical cues were introduced in the places at which it intersected the upper and lower regions of the frame. It is only for stimuli that share this kind of simplicity that we conclude in favor of the contact preservation hypothesis, on account of our findings. We do not claim the unconditional validity of that hypothesis. In fact, it is acknowledged in the literature that the relationship between optical contact (in a 2-D stimulus structure) and perceptual contact (in a cor- 
responding 3-D perceptual organization), when referred to arbitrary visual contexts, cannot be simplified into the direct connection stated by the hypothesis; note the clause "in the absence of information to the contrary" in the definition by Sedgwick, quoted above. In its generality, optical contact is neither a necessary nor a sufficient condition for perceptual contact: It is not necessary, since in cases of partial perceptual occlusion, there may be perceptual continuity in the absence of optical continuity; and it is not sufficient, since by adding suitable cues (e.g., shadows or binocular disparities) in the locus of optical contact of two regions, these regions may perceptually appear to be detached from each other (see Koning \& van Lier, 2003, 2004; Madison, Thompson, Kersten, Shirley, \& Smits, 2001; Ni, Braunstein, \& Andersen, 2004; Saiki \& Hummel, 1998). ${ }^{4}$

Optical tilt $T$ of the target was the second most important stimulus variable in our experiments (after vertical unbalance $V$ ). The remaining comments here regard its effects on the apparent slant of the target. One effect expresses itself as a privileged association of stimulus condition $T=0$ (the target is vertical on the image plane) and perceptual response $S=0$ (the target is judged to have no slant in depth in the perceived scene). This connection may be seen in the results of Experiment 1 (see, e.g., Table 3), which show that the probability of the $S=0$ response is greatly enhanced in the $T=0$ condition, irrespective of varying values of stimulus measure $V$. The same connection is testified by the results of Experiment 2 (see, e.g., Figure 3B). To explain this, we appeal to the generic viewpoint assumption (Freeman, 1994) by considering that if a linear object is not upright in 3-D space, there are only two viewing directions from which its optical image is upright (for an observer standing ordinarily), whereas if the object is upright in 3-D space, its optical image is upright from every viewing direction. Thus, the generic view principle predicts that when the 2-D image of a linear object is upright (i.e., the $T=0$ condition is true), the impression of the object standing vertically in the 3-D space will prevail (i.e., the $S=0$ response becomes highly probable). The effect of the $T=0$ condition on variable $S$, thus interpreted, has something of a veto effect or cue dominance (Bülthoff \& Mallot, 1990, p. 123; Howard \& Rogers, 2002, p. 470): The $T=0$ condition makes the $S=0$ response almost mandatory. This implies that when possible dependence relations of $S$ on stimulus variables other than $T$ are studied (in our research, the dependence of $S$ on vertical unbalance $V$ ), in order to better appreciate such possible relations, the experimenter should include stimulus situations in which $T \neq 0$; that is, the target has nonnull tilt on the image plane.

The designs of our experiments were so defined that, for each $T \neq 0$, there was exact balance in number between stimulus situations in which the $S>0$ response should be favored (by the depth induction or the reverse projection processes) and stimulus situations in which the $S<0$ response should be favored (by the same processes). In spite of this balance in the experimental designs, the experimental results showed an unbalance, which was in favor of the $S>0$ response (upward slant). This can be seen for Experiment 1 in Table 3 (frequency of $S=1$ is greater than frequency of $S=-1$, both for $T=-1$ and for $T=1$ ), and for Experiment 2 in Figure 3B (the diagram is in the upper half-plane). At present, we have no satisfactory explanation for such a bias to upward slant. We have a conjecture, specifically referring to Experiment 2 , in which the bias appeared quite neat (the mean matched slant was positive also in the $T=0$ condition, but lower than that in the $T \neq 0$ condition, presumably due to the privileged association of $T=0$ and $S=0$ ). The conjecture is that the bias obtained in Experiment 2 may also have been due to factors inherent in the special response procedure we used. For evidence on this point, we performed one additional experiment, in which 18 of the 20 participants in Experiment 2 were set in the same conditions, except that the monitor showed no structured stimulus (it was illuminated but empty). The task was to adjust the pole of the response device so as to appear vertical. The values we obtained ranged from $-2.8^{\circ}$ to $9.01^{\circ}$ (on the matched slant scale, measured by the ELITE system), with $M=2.47^{\circ}$ and $S D=2.92^{\circ}$. Thus, we may conclude that there was also an effect conditional on the special setup in Experiment 2, which contributed to determining the upward bias in the data.

Researchers on vision have also considered the possibility that a nonnull tilt of a linear unit in a picture may itself act as a cue to depth (a cue related to linear perspective), thus suggesting an apparent recession in depth of that unit. For example, this assumption is intrinsic in the depth-processing account of the Poggendorff illusion by Gillam (1971) (see also Gillam, 1980, about the MüllerLyer and Ponzo illusions; Koning \& van Lier, 2007). Now, the second effect of the optical tilt of the targetthe effect we called a reverse projection process - may be viewed as an example of this possibility: Optical tilt $T$ is a factor that influences the apparent slant $S$ of the target by interacting with the horizontal position $H$ of the target itself. This effect is proven real both by Experiment 1 (the significance of component $\lambda^{T H S}$ in the log-linear model fitted to the data) and by Experiment 2 (the significance of the interaction effects of factors $T$ and $H$ on variable $S$ ). Since both the depth induction and the reverse projection processes act on perceptual variable $S$, we tried to evaluate their importance comparatively, through supplementary analysis of the data from Experiment 2 (Table 6). The analysis suggested that both processes interact by cumulating their effects on variable $S$ and that the former process (depth induction) appears to be stronger than the latter (reverse projection). Recent studies on depth vision (e.g., He, Wu, Ooi, Yarbrough, \& Wu, 2004; Madison et al., 2001; Ni, Braunstein, \& Andersen, 2004, $2005,2007)$ have examined the interaction of processes relating to optical contact and other factors of perceptual depth, such as occlusion, shadows, and motion parallax. The comparative evaluation we made may be viewed as an item to be added to this set of results, since the depth induction process we discussed essentially involves the optical contact condition. 


\section{AUTHOR NOTE}

Many thanks to Luisa Sartori and Umberto Castiello for instructions concerning the recording apparatus in Experiment 2. We also thank the reviewers of our manuscript, whose suggestions and critical observations were quite valuable for improving our work. Correspondence concerning this article should be addressed to L. Burigana, Dipartimento di Psicologia Generale, Università di Padova, via Venezia 8, I-35131 Padova, Italy (e-mail: luigi.burigana@unipd.it).

\section{REFERENCES}

Agresti, A. (2002). Categorical data analysis. New York: Wiley.

Albert, M. K. (1999). Surface formation and depth in monocular scene perception. Perception, 28, 1347-1360. doi:10.1068/p2987

Bian, Z., Braunstein, M. L., \& Andersen, G. J. (2005). The ground dominance effect in the perception of 3-D layout. Perception \& Psychophysics, 67, 802-815.

Bian, Z., Braunstein, M. L., \& Andersen, G. J. (2006). The ground dominance effect in the perception of relative distance in 3-D scenes is mainly due to characteristics of the ground surface. Perception \& Psychophysics, 68, 1297-1309.

Bülthoff, H. H., \& MaLlot, H. А. (1990). Integration of stereo, shading and texture. In A. Blake \& T. Troscianko (Eds.), AI and the eye (pp. 119-146). Chichester, U.K.: Wiley.

FreEMAN, W. T. (1994). The generic viewpoint assumption in a framework for visual perception. Nature, 368, 542-545. doi:10.1038/368542a0

GiBson, J. J. (1950). The perception of the visual world. Boston: Houghton Mifflin.

Gillam, B. (1971). A depth processing theory of the Poggendorff illusion. Perception \& Psychophysics, 10, 211-216.

Gillam, B. (1980). Geometrical illusions. Scientific American, 242, 87-94.

Gogel, W. C. (1972). Depth adjacency and cue effectiveness. Journal of Experimental Psychology, 92, 176-181.

He, Z. J., Wu, B., OoI, T. L., Yarbrough, G., \& Wu, J. (2004). Judging egocentric distance on the ground: Occlusion and surface integration. Perception, 33, 789-806. doi:10.1068/p5256

Howard, I. P., \& Rogers, B. J. (2002). Seeing in depth: Vol. 2. Depth perception. Toronto: Porteous.

JoHANSSON, G. (1970). On theories for visual space perception: A letter to Gibson. Scandinavian Journal of Psychology, 11, 67-74.

KoffKa, K. (1935). Principles of Gestalt psychology. New York: Harcourt, Brace \& World.

KonING, A., \& VAN LIER, R. (2003). Object-based connectedness facilitates matching. Perception \& Psychophysics, 65, 1094-1102.

Koning, A., \& VAN LIER, R. (2004). Mental rotation depends on the number of objects rather than on the number of image fragments. Acta Psychologica, 117, 65-77. doi:10.1016/j.actpsy.2004.05.002

Koning, A., \& VAN Lier, R. (2005). From interpretation to segmentation. Psychonomic Bulletin \& Review, 12, 917-924.

Koning, A., \& van Lier, R. (2007). 3-D processing in the Poggendorff illusion. Acta Psychologica, 126, 46-58. doi:10.1016/j.actpsy 2006.09.011

Madison, C., Thompson, W., Kersten, D., Shirley, P., \& Smits, B. (2001). Use of interreflection and shadow for surface contact. Perception \& Psychophysics, 63, 187-194.

Meng, J. C., \& Sedgwick, H. A. (2001). Distance perception mediated through nested contact relations among surfaces. Perception \& Psychophysics, 63, 1-15.

Meng, J. C., \& Sedgwick, H. A. (2002). Distance perception across spatial discontinuities. Perception \& Psychophysics, 64, 1-14.

Ni, R., Braunstein, M. L., \& Andersen, G. J. (2004). Perception of scene layout from optical contact, shadows, and motion. Perception, 33, 1305-1318. doi:10.1068/p5288

Ni, R., Braunstein, M. L., \& Andersen, G. J. (2005). Distance percep- tion from motion parallax and ground contact. Visual Cognition, 12, 1235-1254. doi:10.1080/13506280444000724

Ni, R., Braunstein, M. L., \& Andersen, G. J. (2007). Scene layout from ground contact, occlusion, and motion parallax. Visual Cognition, 15, 48-68. doi:10.1080/13506280600646657

ReinhardT-Rutland, A. H. (1999). The framing effect with rectangular and trapezoidal surfaces: Actual and pictorial surface slant, frame orientation, and viewing condition. Perception, 28, 1361-1371. doi: $10.1068 / \mathrm{p} 2950$

Rock, I. (1983). The logic of perception. Cambridge, MA: MIT Press.

SAIKI, J., \& Hummel, J. E. (1998). Connectedness and the integration of parts with relations in shape perception. Journal of Experimental Psychology: Human Perception \& Performance, 24, 227-251. doi:10.1037/0096-1523.24.1.227

Sauer, C. W., Braunstein, M. L., Saidpour, A., \& Andersen, G. J. (2002). Propagation of depth information from local regions in 3-D scenes. Perception, 31, 1047-1059. doi:10.1068/p3261

Sauer, C. W., Saidpour, A., Braunstein, M. L., \& Andersen, G. J. (2001). Perceived depth of 3-D objects in 3-D scenes. Perception, 30, 681-692. doi:10.1068/p3087

SedGwick, H. A. (2001). Visual space perception. In E. B. Goldstein (Ed.), Blackwell handbook of perception (pp. 128-167). Malden, MA: Blackwell.

\section{NOTES}

1. We use the adjective optical when referring to a content (a region, property, relation, etc.) inherent in the pictorial stimulus (the image on the monitor), which is 2-D in character. In the proper sense, the adjective optical refers to the optical image that is projected by a physical reality on the retina. However, if that reality is a pictorial composition (as are the stimuli in our research), a direct correspondence holds true between its contents and the contents in its optical image.

2 . In using the expression optical contact with the stated meaning, we follow Gibson (1950, p. 178), Sedgwick (2001), Bian, Braunstein, and Andersen (2005), and others. The expressions connectedness in the image and image-based connectedness, also used in the psychophysical literature (cf. Koning \& van Lier, 2003; Saiki \& Hummel, 1998), appear to be equivalent in meaning.

3 . There are also differences in the experimental procedure. The participants in Experiments 4 and 5 of Bian et al. (2005) were shown two posts in every stimulus situation, each containing a special dot, and were asked to judge "which of the two dots appeared closer." In these conditions, any conclusion regarding possible apparent slant of the posts should be reached indirectly, on the basis of comparative judgments of apparent egocentric distances of selected particles of the posts. Instead, the participants in our experiments were asked to directly evaluate the apparent slant of the target (the pole in the corridor), either by choosing one of three alternative responses (Experiment 1) or by adjusting a probe pole (Experiment 2)

4. Recent studies have shown that, when operating on a variety of stimuli in which the dichotomy image-based (optical) connectedness versus disconnectedness is made independent of the dichotomy objectbased (perceptual) connectedness versus disconnectedness, it is the latter dichotomy that mostly conditions the performance of participants on certain psychophysical tasks (Koning \& van Lier, 2003, 2004, 2005). These findings gain special meaning when the distinction between the two dichotomies becomes associated with the distinction between an analytic/proximal mode and a normal/world mode in perception (cf. Koffka, 1935, p. 235; Rock, 1983, p. 254). Then they signify that it is the second mode or attitude in perception that is directly relevant to those psychophysical tasks.

(Manuscript received December 17, 2008; revision accepted for publication March 8, 2009.) 